\title{
M. CRAMER
}

\section{Lissage d'un fractile d'une distribution de probabilité variable}

Revue française d'automatique, d'informatique et de recherche opérationnelle. Recherche opérationnelle, tome 8, n V3 (1974), p. 21-31.

<http://www.numdam.org/item?id=RO_1974_8_3_21_0>

(C) AFCET, 1974, tous droits réservés.

L'accès aux archives de la revue «Revue française d'automatique, d'informatique et de recherche opérationnelle. Recherche opérationnelle » implique l'accord avec les conditions générales d'utilisation (http://www.numdam.org/ legal.php). Toute utilisation commerciale ou impression systématique est constitutive d'une infraction pénale. Toute copie ou impression de ce fichier doit contenir la présente mention de copyright.

\section{Numdam}

Article numérisé dans le cadre du programme

Numérisation de documents anciens mathématiques

http://www.numdam.org/ 


\title{
LISSAGE D'UN FRAGTILE D'UNE DISTRIBUTION DE PROBABILITE VARIABLE
}

\author{
par M. CRAmer ( ${ }^{1}$ )
}

Résumé. - Nous exposons le principe d'une nouvelle technique pour estimer un fractile donné d'une variable aléatoire d'après la chronique qu'elle engendre; cette méthode s'adapte à de légères variations de la distribution : à la manière du lissage exponentiel, elle attribue un poids plus grand aux dernières observations. Nous comparons par simulation la performance de cette méthode à celle des techniques courantes utilisées par les programmes industriels de gestion des stocks.

Au préalable, pour situer le cadre pratique de cette étude, nous rappelons la formulation du problème de stock classique et le principe de sa résolution par les programmes spécialisés.

\section{PRINCIPE DE L'ESTIMATION DU FRACTILE CRITIQUE PAR LES PROGRAMMES DE GESTION DES STOCKS}

\section{1-1. Définition du fractile critique}

La gestion économique d'un stock conduit à rechercher le niveau $s$ du stock qui satisfera au moindre coût la demande incertaine $z$. Deux cas sont possibles : excès de stock $(s \geqslant z)$ ou défaut de stock $(s<z)$. On admet souvent que les coûts d'excès et de défaut de stock sont proportionnels aux quantités :

$$
\begin{aligned}
& \text { coût d'excès }=c_{e} \cdot(s-z) \text {, si } s \geqslant z \\
& \text { coût de défaut }=c_{d} \cdot(z-s) \text {, si } s<z
\end{aligned}
$$

Quand on interprète la demande comme une variable aléatoire avec une fonction de répartition continue $F$, la dérivée de l'espérance du coût s'écrit :

$$
c_{e} \cdot F(s)-c_{d} \cdot[1-F(s)]
$$

(1) Centre d'Enseignement Supérieur du Management Public, Arcueil.

Revue Française d'Automatique, Informatique et Recherche Opérationnelle nº nov. 1974, V-3. 
Et comme elle est croissante, le(s) stock(s) $s^{*}$ qui vérifie :

$$
F\left(s^{*}\right)=Q=\frac{c_{d}}{c_{e}+c_{d}},
$$

réalise le coût espéré minimal. Ainsi la caractéristique du stock optimal est d'assurer le service de la demande avec la probabilité $Q$ : c'est le « fractile critique » de la distribution de la demande ([1], [2]).

1-2. Estimation du fractile critique par les programmes industriels ([6], [5])

En pratique l'information se réduit souvent à l'historique des demandes passées : $z_{1}, z_{2}, z_{3}, \ldots z_{t}, \ldots$ Si les coûts mis en jeu sont faibles et le nombre d'articles stockés important, les techniques statistiques de prévision sur chroniques sont écartées au profit de méthodes empiriques moins onéreuses comme la moyenne mobile ou surtout le lissage exponentiel et ses variantes (méthode de Holt; de Winters). La majorité des programmes de gestion des stocks calculent alors le fractile critique à partir d'une prévision de la demande et d'une estimation de la moyenne de l'écart entre cette prévision et l'observation, cela en admettant que cet écart suit une loi normale. Par exemple, quand on emploie le lissage simple ([5]) pour estimer la demande et l'écart, ce calcul du fractile est illustré par les formules élémentaires suivantes :

$$
\begin{array}{ll}
m_{t}=m_{t-1}+a \cdot\left(z_{t-1}-m_{t-1}\right) & (0<a<1) \\
e_{t}=e_{t-1}+b \cdot\left(\left|z_{t-1}-m_{t-1}\right|-e_{t-1}\right) & (0<b<1) \\
s_{t}=m_{t}+k \cdot e_{t} &
\end{array}
$$

où $m_{t}, e_{t}$ et $s_{t}$ sont les estimations courantes de la demande moyenne, de l'écart moyen et du $Q$-fractile, évaluées au temps $t-1 ; k$ est déduit de $Q$ suivant l'hypothèse que $z_{t}-m_{t}$ suit une loi normale d'écart absolu moyen $e_{t}$.

Comme l'objectif essentiel est d'assurer la qualité de service $Q$, qui réalise l'optimum économique, ce procédé, à notre sens, présente deux inconvénients :

indirect, il accumule les erreurs de deux prévisions successives (de plus, comme il est basé sur des estimations de moyennes, il sera souvent trop sensible aux valeurs extrêmes de la demande);

spécialisé, il s'adapte mal aux distributions de demande non normales (son efficacité est particulièrement compromise dans le cas, fréquent en pratique, de distributions discrètes positives de petite moyenne).

Ces considérations nous ont amené à rechercher une technique adaptative pour l'estimation directe du fractile critique. Nous exposons maintenant notre résultat. 


\section{PRINCIPE DU «LISSAGE DE FRACTILE »}

\section{2-1. Définition du lissage de fractile}

Le principe de la méthode que nous préconisons se définit par la formule suivante :

$$
s_{t}=s_{t-1}+c \cdot\left[Q-I\left(s_{t-1} \geqslant z_{t-1}\right)\right]
$$

où :

$I$ est la fonction caractéristique.

$c$ est un coefficient d'adaptativité.

On voit que cette formule s'apparente à un lissage mais elle s'applique directement à l'estimation courante $s_{t-1}$ de $s^{*}$ sans avoir recours à l'approximation normale.

\section{2-2. Propriétés théoriques du lissage de fractile}

Quand les demandes successives $\left(z_{t}\right)$ sont indépendantes et de même distribution $(F)$, l'équation (4) définit un processus markovien stationnaire $\left(s_{t}\right)$ : nous étudions ses propriétés.

\section{2-2.1. Existence d'une distribution invariante}

Nous admettons les hypothèses suivantes, que la pratique justifie :

$s_{0}$ est fixé.

$Q$ est rationnel : $Q=\frac{\alpha}{\alpha+\beta}$, où $\alpha$ et $\beta$ sont des entiers positifs premiers entre eux.

La distribution de la demande a un support borné.

En outre, il est commode de poser :

$$
\begin{aligned}
& c=\alpha+\beta \text { (choix d'unité) } \\
& s_{0}=0 \quad \text { (choix d'origine) }
\end{aligned}
$$

L'état de la chaîne de Markov $\left(s_{t}\right)$ se déplace alors sur la droite entière $Z$, par bonds de $+\alpha$ et de $-\beta$. Ses probabilités de transition se formulent ainsi :

$$
\left\{\begin{array}{l}
p(s, s+\alpha)=1-F(s) \\
p(s, s-\beta)=F(s)
\end{array} \quad(s \in Z)\right.
$$

Étant donné deux entiers quelconques $s^{\prime}$ et $s^{\prime \prime}$, l'arithmétique montre qu'il existe une suite finie de bonds $+\alpha$ et $-\beta$ menant de $s^{\prime}$ à $s^{\prime \prime}$, du fait que $\alpha$ et $\beta$ no novembre 1974, V-3. 
sont premiers entre eux. Il existe en effet des couples d'entiers non négatifs $(\mu, \nu)$ tels que :

$$
\mu \cdot \alpha-\nu \cdot \beta=s^{\prime \prime}-s^{\prime} \quad \text { (corollaire du théorème de Bezout) }
$$

Soit donc $\left(\mu^{*}, \nu^{*}\right)$ le couple vérifiant cette propriété dont la somme $\mu^{*}+\nu^{*}$ est minimale.

Prenons alors $s^{\prime \prime}$ tel que : $F\left(s^{\prime \prime}+1\right)>0$ et $F\left(s^{\prime \prime}-1\right)<1$ et construisons l'arc de trajectoire $\left(x_{n}\right)$ comme suit :

$$
\begin{aligned}
& x_{0}=s^{\prime} \\
& x_{n-1}=\left\{\begin{array}{lll}
x_{n}+\alpha, & \text { si } & x_{n} \leqslant s^{\prime \prime} \\
x_{n}-\beta, & \text { si } & x_{n}>s^{\prime \prime}
\end{array}\right.
\end{aligned}
$$

Notons que les transitions successives sur cet arc ont chacune une probabilité non nulle dans la chaîne $\left(s_{t}\right)$. Désignons par $\mu_{n}$ et $\nu_{n}$ les nombres des deux types de bonds, de $x_{0}$ à $x_{n}$ :

$$
\begin{aligned}
\mu_{n}= & \sum_{k=0}^{k=n-1} I\left(x_{k} \leqslant s^{\prime \prime}\right) \quad \nu_{n}=n-\mu_{n} \\
& x_{n}-s^{\prime}=\mu_{n} \cdot \alpha-v_{n} \cdot \beta
\end{aligned}
$$

Il existe certainement un indice $i$ tel que $: \mu_{i}=\mu^{*}$, et d'après (5) et (7) :

$$
s^{\prime \prime}-x_{i}=\left(s^{\prime \prime}-s^{\prime}\right)-\left(x_{i}-s^{\prime}\right)=-\left(v^{*}-v_{i}\right) \cdot \beta
$$

Montrons par l'absurde que : $v^{*} \geqslant v_{i}$. Si $: \nu^{*}<v_{i}$, alors : $x_{i} \leqslant s^{\prime \prime}-\beta$, ce qui d'après (6) entraîne nécessairement : $x_{i-1}=x_{i}-\alpha$ et par récurrence : $x_{0}=x_{i}-i \cdot \alpha$; d'où la contradiction : $v_{i}=0$. Par conséquent $: \nu^{*} \geqslant v_{i}$, et la trajectoire $\left(x_{k}\right)$ atteint donc $s^{\prime \prime}$ en un nombre de transitions $i+\left(\nu^{*}-v_{i}\right)=\mu^{*}+\nu^{*}:$

$$
x_{\mu^{*}+v^{*}}=s^{\prime \prime}
$$

Cela prouve que, dans la chaîne $\left(s_{t}\right)$, tout état $s^{\prime \prime}$ de l'ensemble : $E_{1}=[s: s \in Z, F(s+1)>0, F(s-1)<1]$, est accessible à partir de tout état $s^{\prime}$ de $Z$.

Remarquons alors que :

$E_{1}$ n'est pas vide.

Les états accessibles depuis $E_{1}$ constituent l'ensemble :

$E_{2}=[s: s \in Z, F(s+\beta)>0, F(s-\alpha)<1]$, qui contient $E_{1}$.

Revue Française d'Automatique, Informatique et Recherche Opérationnelle 
Partant d'un entier extérieur à $E_{2}$, la chaîne atteint $E_{2}$ en un temps fini.

En définitive, cet ensemble fini $E_{2}$ apparaît comme l'unique classe récurrente de la chaîne : cela garantit l'existence d'une distribution invariante unique ([3], [4]).

Cette distribution $P$ exprime le temps relatif d'occupation de chaque état :

$$
\text { Prob. }\left[\lim _{T \rightarrow \infty} \frac{\sum_{t=0}^{T} I\left(s_{t}=s\right)}{T}=P(s)\right]=1
$$

Désignons par $y$ une variable aléatoire distribuée suivant $P$. Si $z$ est une demande aléatoire, alors $y^{\prime}=y+c[Q-I(y \geqslant z)]$ est aussi distribué suivant $P$, si bien que : $E[F(y)]=Q$; la moyenne à long terme de $F\left(s_{t}\right)$ est donc égale à $Q$.

Étant donné $F$, on peut calculer $P$ à partir des équations :

$$
P(s)=[1-F(s-\alpha)] P(s-\alpha)+F(s+\beta) P(s+\beta) \quad\left[P\left(E_{2}\right)=1\right]
$$

Quand : $\alpha=\beta=1$ et $E_{2}=(0,1,2, \ldots N), P$ se formule :

$$
P(s)=P(O) \cdot \prod_{n=0}^{n=s-1} \frac{1-F(n)}{F(n+1)} \quad(s=1,2, \ldots N)
$$

En particulier, si la distribution $(F)$ est uniforme, la loi $P$ est binomiale :

$$
P(s)=2^{-N} C_{N}^{s} \quad(s=0,1, \ldots N)
$$

et le calcul donne $: \operatorname{var}[F(y)]=\frac{1}{4 N}$, soit $\frac{c}{8 N}$ dans le cas général où $\frac{c}{2} \mathrm{n}$ 'est pas l'unité; en diminuant $c$, on peut donc rendre l'estimateur $\left(s_{t}\right)$ aussi précis que l'on veut.

Comme les conditions simplificatrices : $c=\alpha+\beta, s_{0}=0$, ne sont pas nécessaires à l'existence de $P$, nous ne les retenons pas par la suite.

\section{2-2.2. Une borne pour l'erreur sur le fractile à long terme}

Définissons :

$s^{*}$, tel que $F\left(s^{*}\right)=Q$.

$\pi=F\left[s^{*}+c(1-Q)\right]-F\left(s^{*}-c Q\right)$.

$y$ distribué suivant $P$, et une demande aléatoire $z$.

$v=\left|y-s^{*}\right|-\left|y+c[Q-I(y \geqslant z)]-s^{*}\right|$.

$u=c|Q-F(y)|-E(v \mid y)$.

$\mathrm{n}^{\circ}$ novembre $1974, \mathrm{~V}-3$. 
On peut calculer la forme de $u$ suivant la valeur de $y$ :

$$
\begin{gathered}
y \leqslant s^{*}-c Q \text { ou } y \geqslant s^{*}+c(1-Q) \Rightarrow u=0 . \\
s^{*}-c Q<y<s^{*} \Rightarrow u=2[1-F(y)]\left(y-s^{*}+c Q\right) \leqslant 2[1-(Q-\pi)] \cdot c Q . \\
s^{*} \leqslant y<s^{*}+c(1-Q) \Rightarrow u=2 F(y)\left[s^{*}-y+c(1-Q)\right] \leqslant 2(Q+\pi) \cdot c(1-Q) .
\end{gathered}
$$

Comme $E v=0$ en raison de l'invariance de $P$, nous en déduisons la borne suivante :

$$
E[|Q-F(y)|] \leqslant 2[Q(1-Q)+\pi] \cdot P\{] s^{*}-c Q, s^{*}+c(1-Q)[\}
$$

Notons que :

$$
Q(1-Q)<1 / 4
$$

Si $F$ est continue $: \pi \rightarrow 0$ quand $c \rightarrow 0$.

Si $F$ a une dérivée $F^{\prime}: \pi \leqslant c \cdot \sup \left(F^{\prime}\right)$.

En pratique, pour que l'estimation du fractile $s^{*}$ ait une précision satisfaisante, $c$ sera choisi petit par rapport au domaine de $z$, et $\pi$ sera négligeable devant $Q(1-Q)$. Dès lors, si l'erreur à long terme sur le fractile : $E^{*}=E[|Q-F(y)|], \quad$ reste importante, c'est que : $P^{*}=P\{] s^{*}-c Q$, $s^{*}+c(1-Q)[\}$, a une valeur particulièrement forte, donc que $\left(s_{t}\right)$ est néanmoins souvent voisin de $s^{*}$. Par exemple :

$$
\begin{aligned}
& Q=0,95 \quad E^{*}=0,05 \Rightarrow P^{*}>0,52 . \\
& Q=0,99 \Rightarrow E^{*}<0,02 .
\end{aligned}
$$

\section{2-2.3. Une formule approchée pour la distribution invariante}

Afin de calculer une formule approchée de $P$ dans le cas général, nous. admettons que $P$ a une densité $p$, et que $F$ et $p$ ont des dérivées secondes.

Partant de l'équation d'invariance :

$$
P(s)=[1-F(s-c Q)] \cdot p(s-c Q)+F[s+c(1-Q)] \cdot p[s+c(1-Q)],
$$

nous développons alors $F$ et $p$ jusqu'au second ordre :

$$
\begin{aligned}
p(s)=[1-F(s)+ & \left.c Q F^{\prime}(s)-\frac{c^{2} Q^{2}}{2} F^{\prime \prime}(s)\right] \cdot\left[p(s)-c Q p^{\prime}(s)+\frac{c^{2} Q^{2}}{2} p^{\prime \prime}(s)\right] \\
+ & {\left[F(s)+c(1-Q) F^{\prime}(s)+\frac{c^{2}(1-Q)^{2}}{2} F^{\prime \prime}(s)\right] } \\
\cdot & {\left[p(s)+c(1-Q) p^{\prime}(s)+\frac{c^{2}(1-Q)^{2}}{2} p^{\prime \prime}(s)\right]+c^{2} \cdot O(c) . }
\end{aligned}
$$


D'où, après simplification :

$$
\begin{aligned}
O=c F^{\prime} p+c(F-Q) p^{\prime} & +\frac{c^{2}}{2}\left[(1-Q)^{2}-Q^{2}\right] F^{\prime \prime} p \\
& +c^{2}\left[(1-Q)^{2}-Q^{2}\right] F^{\prime} p^{\prime} \\
& +\frac{c^{2}}{2}\left[Q^{2}(1-F)+(1-Q)^{2} F\right] p^{\prime \prime}+c^{2} \cdot O(c) .
\end{aligned}
$$

Négligeons le dernier terme et intégrons deux fois :

(10) $(F-Q) p+\frac{c}{2}\left[(1-Q)^{2}-Q^{2}\right] F^{\prime} p$

$$
+\frac{c}{2}\left[Q^{2}(1-F)+(1-Q)^{2} F\right] p^{\prime}=A_{1} \quad\left(A_{1} \text { constant }\right)
$$

(11) $\int_{s^{*}}^{s}[F(x)-Q] p(x) \mathrm{d} x$

$$
+\frac{c}{2}\left[Q^{2}(1-F(s))+(1-Q)^{2} F(s)\right] p(s)=A_{1} s+A_{2} \quad\left(A_{2} \text { const. }\right)
$$

D'après (11), $A_{1}$ est nul car sinon : $\lim _{s \rightarrow \infty} p(s)=\infty$; nous pouvons donc tirer $\frac{p^{\prime}}{p}$ de $(10)$ :

$$
\begin{gathered}
\frac{p^{\prime}}{p}=-\frac{2}{c} \cdot \frac{F-Q}{Q^{2}(1-F)+(1-Q)^{2} F}-\frac{\left[(1-Q)^{2}-Q^{2}\right] F^{\prime}}{Q^{2}(1-F)+(1-Q)^{2} F} \Rightarrow \\
p(s)=\frac{B}{H(s)} \cdot c^{-\frac{2}{c} \int_{s}^{s} \frac{F(x)-Q}{H(x)} \cdot \mathrm{d} x}
\end{gathered}
$$

avec : $H(x)=Q^{2}[1-F(x)]+(1-Q)^{2} F(x)$ et $B=p\left(s^{*}\right) H\left(s^{*}\right)=\frac{2}{c} A_{2}$.

Pour $Q=1 / 2$, (12) se simplifie car $H=1 / 4$ :

$$
p(s)=p\left(s^{*}\right) \cdot \mathrm{e}^{-\frac{8}{c} \int_{i}^{s}[F(x)-Q] \mathrm{d} x}
$$

En particulier, si $F$ a une densité uniforme $d$ sur l'intervalle $U$ contenant $s^{*}$, $p$ suit une loi normale sur $U$ :

$$
\left.p(s)=p\left(s^{*}\right) \cdot \mathrm{e}^{-\frac{4 d}{c} \cdot\left(s-s^{*}\right)^{2}} \quad s \in U\right)
$$

(Ce résultat est l'expression asymptotique de (8) quand $\frac{c}{N} \rightarrow 0$ ). $n^{\circ}$ novembre $1974, \mathrm{~V}-3$. 
On voit que ces diverses formules [(12), (13), (14)] indiquent que $p(s)$ décroît d'une manière exponentielle quand $F(s)$ s'éloigne de $Q$.

Par exemple, dans le cas : $Q=\frac{1}{2}$, prenons $\bar{s}$ tel que : $F(s)=\frac{1}{2}+\eta(\eta>0)$ : alors, d'après (13) :

$$
s \geqslant \bar{s} \quad p(s) \leqslant p(s) \cdot \mathrm{e}^{-\frac{8 \eta}{c} \cdot(s-\bar{s})}
$$

\section{COMPARAISON DES DEUX TECHNIQUES PAR SIMULATION}

Nous avons placé en compétition :

. le lissage classique, représenté par les formules (1), (2), (3),

. le lissage de fractile, représenté par la formule (4),

en les appliquant à diverses lois de probabilité (normale, poissonnienne, binomiale, uniforme, exponentielle).

En général, le lissage de fractile, malgré son calcul restreint et son unique paramètre, fournit une estimation plus précise du fractile recherché que le lissage classique.

A titre d'illustration, nous donnons les résultats de ces simulations dans deux cas particuliers :

. l'estimation de la médiane d'une loi normale

[c'est la condition la plus avantageuse pour le lissage classique qui effectue alors une estimation directe par la formule (1)],

. l'estimation du fractile $(0,9)$ d'une loi exponentielle.

\section{3-1. Simulation de l'estimation de la médiane d'une loi normale}

\section{3-1.1. Conditions de la simulation}

$z_{t}$ est distribué suivant une loi normale de moyenne 0 est de variance 1 ; $m_{0}$ [(formule (1)] et $s_{0}$ [formule (4)] portent une même erreur initiale $d_{0}$ :

$$
m_{0}=s_{0}=d_{0}
$$

Le critère d'évaluation est la somme des valeurs absolues des écarts :

$$
\begin{aligned}
& \left|m_{t}-z_{t}\right| \text { pour le lissage classique } \\
& \left|s_{t}-z_{t}\right| \text { pour le lissage de fractile }
\end{aligned}
$$

sur un horizon de $T$ périodes $(t=1,2, \ldots T)$. 
3-1.2. Résultats

\begin{tabular}{|c|c|c|c|c|c|c|c|c|}
\hline \multirow[b]{3}{*}{ 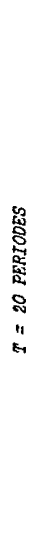 } & \multicolumn{4}{|c|}{ Lissage Classique } & \multicolumn{4}{|c|}{ Lissage de Fractile } \\
\hline & 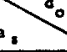 & o & 0,5 & 1 & & 0 & 0,5 & 1 \\
\hline & $\begin{array}{l}0 \\
0,02 \\
0,04 \\
0,06 \\
0,08 \\
0,1 \\
0,12 \\
0,14 \\
0,16 \\
0,18 \\
0,2 \\
0,22 \\
0,24 \\
0,26 \\
0,28 \\
0,3 \\
0,32 \\
0,34 \\
0,36 \\
0,38 \\
0,4\end{array}$ & $\begin{array}{l}8,23 \\
8,30 \\
8,36 \\
8,40 \\
8,43 \\
8,47 \\
8,50 \\
8,53 \\
8,56 \\
8,60 \\
8,64 \\
8,69 \\
8,73 \\
8,78 \\
8,82 \\
8,87 \\
8,91 \\
8,95 \\
8,99 \\
9,02 \\
9,06\end{array}$ & $\begin{array}{r}11,36 \\
10,50 \\
9,99 \\
9,65 \\
9,40 \\
9,24 \\
9,15 \\
9,08 \\
9,06 \\
+9,05 \\
9,06 \\
9,07 \\
9,10 \\
9,13 \\
9,16 \\
9,19 \\
9,22 \\
9,25 \\
9,28 \\
9,31 \\
9,34\end{array}$ & $\begin{array}{l}19,33 \\
16,56 \\
14,54 \\
13,15 \\
12,31 \\
11,7 \\
11,20 \\
10,86 \\
10,62 \\
10,45 \\
10,31 \\
10,21 \\
10,14 \\
10,08 \\
10,04 \\
10,02 \\
10,01 \\
10,01 \\
10,02 \\
10,03 \\
10,04\end{array}$ & $\begin{array}{l}0 \\
0,025 \\
0,05 \\
0,075 \\
0,1 \\
0,125 \\
0,15 \\
0,175 \\
0,2 \\
0,225 \\
0,25 \\
0,275 \\
0,3 \\
0,325 \\
0,35 \\
0,375 \\
0,4 \\
0,425 \\
0,45 \\
0,475 \\
0,5\end{array}$ & $\begin{array}{l}8,23 \\
8,32 \\
8,42 \\
8,52 \\
8,57 \\
8,64 \\
8,75 \\
8,84 \\
8,96 \\
8,95 \\
8,94 \\
9,06 \\
9,18 \\
9,23 \\
9,19 \\
9,31 \\
9,41 \\
9,38 \\
9,50 \\
9,54 \\
9,66\end{array}$ & $\begin{array}{r}11,36 \\
10,57 \\
10,10 \\
9,79 \\
9,58 \\
9,42 \\
9,27 \\
9,33 \\
9,29 \\
9,33 \\
+9,26 \\
9,53 \\
9,51 \\
9,57 \\
9,37 \\
9,33 \\
9,51 \\
9,57 \\
9,69 \\
9,71 \\
9,91\end{array}$ & $\begin{array}{r}19,33 \\
17,38 \\
15,66 \\
14,25 \\
13,39 \\
12,67 \\
12,09 \\
11,72 \\
11,39 \\
11,98 \\
10,96 \\
10,93 \\
10,96 \\
10,8 ! \\
10,70 \\
10,74 \\
10,65 \\
+10,62 \\
10,67 \\
10,66 \\
10,74\end{array}$ \\
\hline & I & $\mathbf{0}$ & 0,5 & 1 & $c$. & 0 & 0,5 & 1 \\
\hline 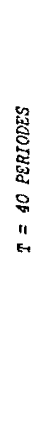 & $\begin{array}{l}0 \\
0,02 \\
0,04 \\
0,06 \\
0,08 \\
0,1 \\
0,12 \\
0,14 \\
0,16 \\
0,18 \\
0,2 \\
0,22 \\
0,24 \\
0,26 \\
0,28 \\
0,3 \\
0,32 \\
0,34 \\
0,36 \\
0,38 \\
0,4\end{array}$ & $\begin{array}{l}16,30 \\
16,36 \\
16,42 \\
16,47 \\
16,53 \\
16,61 \\
16,71 \\
16,81 \\
16,92 \\
17,04 \\
17,16 \\
17,29 \\
17,42 \\
17,55 \\
17,67 \\
17,80 \\
17,93 \\
18,05 \\
18,18 \\
18,30 \\
18,42\end{array}$ & $\begin{array}{l}22,84 \\
19,66 \\
18,4 \\
17,84 \\
17,54 \\
17,40 \\
17,35 \\
17,36 \\
17,40 \\
17,48 \\
17,57 \\
17,68 \\
17,79 \\
17,89 \\
18,00 \\
18,12 \\
18,24 \\
18,35 \\
18,47 \\
18,58 \\
18,7\end{array}$ & $\begin{array}{r}39,43 \\
29,13 \\
24,07 \\
21,66 \\
20,55 \\
19,88 \\
19,40 \\
19,12 \\
18,96 \\
18,88 \\
18,83 \\
+18,81 \\
18,82 \\
18,84 \\
18,89 \\
18,96 \\
19,03 \\
19,11 \\
19,20 \\
19,30 \\
19,39\end{array}$ & $\begin{array}{l}0 \\
0,025 \\
0,05 \\
0,075 \\
0,1 \\
0,125 \\
0,15 \\
0,175 \\
0,2 \\
0,225 \\
0,25 \\
0,275 \\
0,3 \\
0,325 \\
0,35 \\
0,375 \\
0,4 \\
0,425 \\
0,45 \\
0,475 \\
0,5\end{array}$ & $\begin{array}{l}16,30 \\
16,44 \\
16,63 \\
16,83 \\
16,91 \\
17,07 \\
17,24 \\
17,26 \\
17,45 \\
17,54 \\
17,62 \\
17,85 \\
18,04 \\
18,24 \\
18,18 \\
18,49 \\
18,71 \\
18,81 \\
19,06 \\
19,15 \\
19,25\end{array}$ & $\begin{array}{r}22,84 \\
19,94 \\
18,66 \\
18,14 \\
17,91 \\
17,81 \\
+37,60 \\
17,83 \\
17,87 \\
17,80 \\
17,93 \\
18,28 \\
18,37 \\
18,41 \\
18,39 \\
18,45 \\
18,78 \\
18,92 \\
19,15 \\
19,32 \\
19,50\end{array}$ & $\begin{array}{r}39,43 \\
31,63 \\
26,16 \\
23,28 \\
22,00 \\
21,18 \\
20,41 \\
20,21 \\
19,94 \\
19,84 \\
+19,64 \\
19,74 \\
19,71 \\
19,72 \\
19,80 \\
19,71 \\
19,74 \\
19,89 \\
20,10 \\
20,26 \\
20,31\end{array}$ \\
\hline
\end{tabular}

3-2. Simulation de l'estimation du fractile 0,9 d'une loi exponentielle

\section{3-2.1. Conditions de la simulation}

$z_{t}$ est distribué suivant une loi exponentielle de moyenne 1 ; les deux estimations comportent la même erreur à l'origine :

$$
s_{0}=1\left(m_{0}=0,4 ; k \cdot e_{0}=0,6\right)
$$

le critère pour les deux estimateurs est la somme des coûts $c_{t}$ :

$$
\begin{array}{llll}
c_{t}=0,9 \cdot\left(z_{t}-s_{t}\right) & \text { si } & z_{t}>s_{t} \\
c_{t}=0,1 \cdot\left(s_{t}-z_{t}\right) & \text { si } & z_{t}<s_{t}
\end{array}
$$




\section{3-2.2. Résultats}

\begin{tabular}{|c|c|c|c|c|c|c|c|c|c|c|c|c|c|c|c|}
\hline \multirow[b]{3}{*}{ 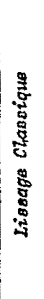 } & \multicolumn{7}{|c|}{$T=20$ PERTODES } & \multicolumn{8}{|c|}{$T=40$ FERTODES } \\
\hline & a. $\quad b=$ & 0 & 0,02 & 0,04 & 0,06 & 0,08 & 1 & $a_{a} \quad b=$ & 0 & 0,02 & 0 , & & 0,06 & 0,08 & a \\
\hline & $\begin{array}{l}0 \\
0,05 \\
0,1 \\
0,15 \\
0,2 \\
0,25 \\
0,3 \\
0,35 \\
0,4\end{array}$ & $\begin{array}{r}5,57 \\
5,02 \\
4,71 \\
4,63 \\
+4,62 \\
4,65 \\
4,71 \\
4,78 \\
4,87\end{array}$ & $\begin{array}{l}5,52 \\
4,94 \\
4,69 \\
4,62 \\
4,53 \\
4,68 \\
4,75 \\
4,84 \\
4,94\end{array}$ & $\begin{array}{l}5,32 \\
4,89 \\
4,67 \\
4,62 \\
4,64 \\
4,70 \\
4,79 \\
4,88 \\
4,99\end{array}$ & $\begin{array}{l}5,24 \\
4,84 \\
4,66 \\
4,63 \\
4,66 \\
4,73 \\
4,82 \\
4,92 \\
5,04\end{array}$ & $\begin{array}{l}5,17 \\
4,81 \\
4,66 \\
4,63 \\
4,67 \\
4,75 \\
4,85 \\
4,96 \\
5,08\end{array}$ & $\begin{array}{l}5,13 \\
4,79 \\
4,67 \\
4,64 \\
4,69 \\
4,77 \\
4,88 \\
4,99 \\
5,12\end{array}$ & $\begin{array}{l}0 \\
0,05 \\
0,1 \\
0,15 \\
0,2 \\
0,25 \\
0,3 \\
0,35 \\
0,4\end{array}$ & $\begin{array}{r}11,51 \\
9,96 \\
9,61 \\
9,59 \\
9,65 \\
9,77 \\
9,90 \\
10,04 \\
10,18\end{array}$ & $\begin{array}{r}10,70 \\
9,76 \\
9,50 \\
9,51 \\
9,60 \\
9,72 \\
9,89 \\
10,07 \\
10,25\end{array}$ & $\begin{array}{r}10 \\
9 \\
9 \\
9 \\
9 \\
9 \\
9 \\
10 \\
10\end{array}$ & & $\begin{array}{r}10,22 \\
9,60 \\
9,41 \\
9,46 \\
9,59 \\
9,74 \\
9,92 \\
10,13 \\
10,36\end{array}$ & $\begin{array}{r}10,13 \\
9,58 \\
-9,41 \\
9,47 \\
9,60 \\
9,77 \\
9,95 \\
10,17 \\
10,41\end{array}$ & $\left\{\begin{array}{r}10,09 \\
9,57 \\
9,43 \\
9,49 \\
9,63 \\
9,80 \\
9,99 \\
10,22 \\
10,47\end{array}\right.$ \\
\hline 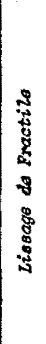 & $\begin{array}{c}c=0 \\
0,1 \\
0,1 \\
0,1 \\
0,1 \\
0,1 \\
0,1 \\
0,1 \\
0,1 \\
0,1 \\
0,1 \\
0,2 \\
0,2 \\
0,2 \\
0,2 \\
0,2 \\
0,2\end{array}$ & $\begin{array}{l}5, \\
5, \\
4, \\
4, \\
4, \\
4, \\
4, \\
4, \\
4, \\
4, \\
4, \\
4, \\
4, \\
4, \\
4, \\
4, \\
4,\end{array}$ & & $\begin{array}{l}0,26 \\
0,27 \\
0,28 \\
0,29 \\
0,3 \\
0,31 \\
0,32 \\
0,33 \\
0,34 \\
0,35 \\
0,36 \\
0,37 \\
0,38 \\
0,39 \\
0,4 \\
0,41 \\
0,42\end{array}$ & $\begin{array}{l}4,64 \\
4,61 \\
4,59 \\
4,56 \\
4,54 \\
4,52 \\
4,49 \\
4,47 \\
4,44 \\
4,42 \\
4,40 \\
4,37 \\
4,35 \\
4,32 \\
4,30 \\
4,28 \\
4,25\end{array}$ & $\begin{array}{c}c=0,43 \\
0,44 \\
0,45 \\
0,46 \\
0,47 \\
0,48 \\
0,49 \\
0,5 \\
0,51 \\
0,52 \\
0,53 \\
0,54 \\
0,55 \\
0,56 \\
0,57 \\
0,58 \\
0,59\end{array}$ & $\begin{array}{r}4,23 \\
4,20 \\
4,18 \\
4,16 \\
4,13 \\
+4,11 \\
4,68 \\
4,67 \\
4,67 \\
4,67 \\
4,66 \\
4,66 \\
4,66 \\
4,66 \\
4,65 \\
4,65 \\
4,65\end{array}$ & $\begin{array}{c}c=0 \\
0,1 \\
0,11 \\
0,12 \\
0,13 \\
0,114 \\
0,15 \\
0,16 \\
0,17 \\
0,18 \\
0,19 \\
0,2 \\
0,21 \\
0,22 \\
0,23 \\
0,24 \\
0,25\end{array}$ & $\begin{array}{r}11,51 \\
9,88 \\
9,79 \\
9,71 \\
9,62 \\
9,53 \\
9,45 \\
9,36 \\
9,68 \\
9,63 \\
9,59 \\
9,54 \\
9,49 \\
9,45 \\
9,40 \\
9,36 \\
9,31\end{array}$ & $\begin{array}{l}0 \\
0 \\
0 \\
0 \\
0 \\
0 \\
0 \\
0 \\
0 \\
0 \\
0\end{array}$ & $\begin{array}{l}26 \\
27 \\
28 \\
29 \\
3 \\
31 \\
32 \\
33 \\
34 \\
35 \\
36 \\
37 \\
38 \\
39 \\
4 \\
41 \\
42\end{array}$ & $\begin{array}{l}9,2 \\
9,2 \\
9,1 \\
9,1 \\
9,0 \\
9,0 \\
8,9 \\
8,9 \\
8,9 \\
8,8 \\
8,8 \\
8,7 \\
8,7 \\
8,6 \\
8,6 \\
8,5 \\
8,5\end{array}$ & & $\begin{array}{l}0,43 \\
0,44 \\
0,45 \\
0,46 \\
0,47 \\
0,43 \\
0,49 \\
0,5 \\
0,51 \\
0,52 \\
0,53 \\
0,54 \\
0,55 \\
0,56 \\
0,57 \\
0,58 \\
0,59\end{array}$ & $\begin{array}{l}8,58 \\
8,44 \\
8,39 \\
8,34 \\
8,30 \\
8,25 \\
9,29 \\
9,28 \\
9,26 \\
9,25 \\
9,23 \\
9,22 \\
9,20 \\
9,19 \\
9,17 \\
9,16 \\
9,14\end{array}$ \\
\hline
\end{tabular}

\section{EXTENSIONS DU LISSAGE DE FRACTILE}

Le lissage de fractile apparaît donc comme une technique a priori efficace pour réduire les coûts d'excès et de défaut, et pour régler la qualité du service. La formule (4), qui exprime le principe de cette technique, peut être adaptée à des applications variées; nous présentons ci-dessous quelques exemples de versions spécialisées du lissage de fractile.

\section{4-1. Forme multiplicative du lissage de fractile}

Quand $\left(z_{t}\right)$ demeure positif et que son aléa (variance) varie dans le même rapport que sa moyenne, il est rationnel d'adopter la variante multiplicative de (4) :

$$
s_{t}=s_{t-1}\left(1+c \cdot\left(Q-I\left(s_{t-1} \geqslant z_{t-1}\right)\right)\right)
$$

(Le coefficient $c$, s'interprétant alors en pourcentage, devient plus facile à régler.)

\subsection{Cas d'une demande-somme de composition variable}

Quand $\left(z_{t}\right)$ est modélisé par une somme de variables aléatoires indépendantes $\left(x_{t}\right)$ sur un ensemble d'indices $J(t)$ variable en fonction de $t$ :

$$
z_{t}=\sum_{i \in J(t)} x_{t}^{i}
$$

Revue Française d'Automatique, Informatique et Recherche Opérationnelle 
on appliquera la formulation suivante :

$$
\begin{aligned}
& s_{t}^{i}=s_{t-1}^{i}+c^{i} \cdot\left(Q-I\left(s_{t-1} \geqslant z_{t-1}\right)\right) \text { pour } i \in J(t-1) \\
& s_{t}=\sum_{i \in j(t)} s_{t}^{i}
\end{aligned}
$$

(On pourra traiter ainsi les effets saisonniers additifs et, en général, tous les facteurs répétitifs dont l'occurence est prévisible.)

\section{4-3. Cas d'un coût de défaut constant (cf. 1-1.)}

Quand le coût de défaut $C_{d}$ est indépendant de la quantité en défaut, la solution de : $c_{e} \cdot F(s)=C_{d} \cdot f(s)$ (où $f$ est la densité de $F$ ), si elle est unique, donne le stock optimal $s^{*}([1])$. Par un raisonnement analogue à celui de 2-2., on en déduit alors que :

$$
s_{t}=s_{t-1}\left\{\begin{array}{lll}
-c \cdot c_{e} & \text { si } & s_{t-1} \geqslant z_{t-1} \\
+C_{c} & \text { si } & s_{t-1}<z_{t-1} \leqslant s_{t-1}+c
\end{array}\right.
$$

est un estimateur de $s^{*}$ dont la précision et l'adaptativité dépendent de $c$.

\section{REMERCIEMENTS}

Pour conclure, qu'il me soit permis de remercier M. NevEu pour ses conseils essentiels sur la théorie, M. TReguer (CESMAP) pour l'élaboration de la simulation et $M^{\text {lle }}$ DuBols (CESMAP) pour l'exécution typographique.

\section{RÉFÉRENCES}

[1] StARr M. K. et Miller D. M., La gestion des stocks : théorie et pratique (Chapitre II.6-7 : notion de fractile critique), Dunod, Paris, 1966.

[2] BuchaU J. et Kanigsberg E., Gestion scientifique des stocks (Chapitre XIX : notion de fractile critique), Les Éditions d'Organisation, Paris, 1965.

[3] KARLIN S., Initiation aux processus aléatoires (Chapitre III : théorèmes limites des chaînes de Markov), Dunod, Paris, 1969.

[4] Dooв J. L., Stochastic processes (Chapitre V : théorèmes limites des chaînes de Markov), Wiley, New-York, 1953.

[5] Brown R. G., Smoothing, Forecasting and Prediction of discrete time series (Lissage exponentiel), Englewood Cliffs, Prentice Hall, 1963.

[6] BARANGer Pierre, Comment réussir votre gestion des stocks sur ordinateur (Annexe 1 : programmes industriels), Les Éditions d'Organisation, Paris, 1972.

[7] RADHAKRISHNA RAO C., Linear statistical inference and its applications (pp. 422-5. statistiques d'ordre), Wiley, New-York, 1965, 